\title{
Correlations between axial and lateral emission of coupled quantum dot-micropillar cavities
}

\author{
A. Musiał, ${ }^{1,2}$ C. Hopfmann, ${ }^{1}$ T. Heindel, ${ }^{1}$ C. Gies,${ }^{3}$ M. Florian, ${ }^{3}$ H. A. M. Leymann,,${ }^{4}$ A. Foerster, ${ }^{4}$ C. Schneider, ${ }^{5}$ \\ F. Jahnke, ${ }^{3}$ S. Höfling, ${ }^{5,6}$ M. Kamp, ${ }^{5}$ and S. Reitzenstein ${ }^{1, *}$ \\ ${ }^{1}$ Institut für Festkörperphysik, Technische Universität Berlin, Hardenbergstraße 36, D-10623 Berlin, Germany \\ ${ }^{2}$ Laboratory for Optical Spectroscopy of Nanostructures, Department of Experimental Physics, Wroctaw University of Technology, \\ Wybrzeże Wyspiańskiego 27, 50-370 Wroctaw, Poland \\ ${ }^{3}$ Institute for Theoretical Physics, University of Bremen, Otto-Hahn-Allee 1, D-28334 Bremen, Germany \\ ${ }^{4}$ Institute for Theoretical Physics, University of Magdeburg, Postfach 4120, D-39016 Magdeburg, Germany \\ ${ }^{5}$ Technische Physik, Physikalisches Institut and Wilhelm Conrad Röntgen-Research Center for Complex Material Systems, \\ Universität Würzburg, Am Hubland, D-97074 Würzburg, Germany \\ ${ }^{6}$ SUPA, School of Physics and Astronomy, University of St. Andrews, St Andrews, KY16 9SS, United Kingdom
}

(Received 15 February 2015; revised manuscript received 30 April 2015; published 19 May 2015)

\begin{abstract}
We report on optical studies of coupled quantum dot-micropillar cavities using a $90^{\circ}$ excitation-and-detection scheme. This specific configuration allows us to excite the micropillar structures either in the axial direction or in the lateral direction and to simultaneously detect emission from both directions. That enables us to reveal correlations between emission into the cavity mode and the leaky modes in the regime of cavity quantum electrodynamics. In particular, we can access and distinguish between axial cavity emission and lateral emission consisting of emission of quantum dots into the leaky modes and losses due to sidewall scattering, respectively. In the multiemitter regime, this technique provides direct access to the respective loss channels and reveals a strong increase of sidewall losses in the low-diameter regime below about $3.0 \mu \mathrm{m}$. Beyond that, in the single-emitter regime, we observe an anticorrelation between quantum dot emission coupled into the cavity mode and into the leaky modes which is controlled by light-matter interaction in the weak coupling regime. This anticorrelation is absent in the strong coupling regime due to the presence of entangled light-matter states. Moreover, excitation-power-dependent studies demonstrate that the intensity ratio between axial and lateral emission increases strongly above the lasing threshold due to enhanced directionality of emission into the lasing mode. In fact, theoretical studies confirm that this intensity ratio is an additional indicator of laser action in high- $\beta$ microlasers for which the onset of lasing is difficult to identify by the input-output characteristics.
\end{abstract}

DOI: 10.1103/PhysRevB.91.205310

PACS number(s): 78.55.Cr, 78.67.Hc, 42.50.Pq, 81.07.Ta

\section{INTRODUCTION}

Quantum dot-(QD) microcavities are excellent candidates to study and utilize light-matter interaction-related phenomena in the framework of cavity quantum electrodynamics (cQED) [1-4]. Achievements in this exciting and very active field of quantum optics in solid-state include numerous studies of cQED on a single QD level in the weak and strong lightmatter coupling regime [5-9]. Possible applications range from nonclassical light sources, such as efficient sources of single photons $[10,11]$ or entangled photon pairs [12], to high- $\beta$ microlasers [1,13-18]. These nanophotonics devices rely on the strong localization of the light field in low mode volume microcavities with high quality $(Q)$ factors and the control of the spontaneous emission process via cQED effects.

Further development of structures relying on pronounced cQED effects requires a profound knowledge about dissipative decay channels, as light confinement and optical losses depend sensitively on the geometry of the microresonator [19]. In the case of micropillar cavities, the dominating loss channels are (i) intentional losses, i.e., outcoupling of light through the upper distributed Bragg reflector (DBR); (ii) intrinsic lateral losses present even in the ideal cavity without any structural imperfections; (iii) scattering losses at the sidewalls; and (iv) absorption in the active layer or in the mirror sections.

\footnotetext{
*stephan.reitzenstein@physik.tu-berlin.de
}

Lateral losses can be reduced by optimizing the fabrication process and the sample layout, e.g., via adiabatic cavity designs [20-22]. This is of particular importance in the case of small-diameter micropillars featuring large light-matter coupling constants due to small mode volumes [19,21,23] and, at the same time, suffering from higher lateral losses due to higher mode intensities at the surface. Unfortunately, lateral losses are not directly accessible in a standard detection geometry normal to the sample's surface, i.e., in the axial direction of the micropillars, in which light is coupled out preferentially through the upper DBR of the micropillar. In this configuration lateral losses can be described only indirectly via their influence on the $Q$ factor of the cavity. Monitoring the emission in the lateral direction, on the other hand, enables direct insight into the lateral-loss channels.

In addition to the outcoupling of the light itself, the spontaneous emission coupling factor $\beta$ plays a crucial role for microcavity-based nonclassical light sources and microlasers, as it determines the photon extraction efficiency and the threshold pump-power density, respectively [24-26]. In QDmicrocavity systems, the $\beta$ factor is usually related to the Purcell factor $F_{P}$ via $\beta=F_{P} /\left(F_{P}+1\right)$ and describes the fraction of spontaneous emission coupled into the cavity mode or-on the single photon level-the probability of coupling a spontaneously emitted photon into the cavity mode. Alternatively, emission is coupled into the leaky modes which should be suppressed to ensure maximum performance of nonclassical light sources and microlasers. To obtain direct 
access to emission into leaky modes, monitoring also lateral emission from QD-micropillar cavities is highly desirable. In particular, this allows one to quantify photon losses via the leaky modes and to probe the directionality of the spontaneous emission pattern, which has not been possible in standard microphotoluminescence ( $\mu \mathrm{PL}$ ) configurations used until now. In this way, important insight into the emission process can be gained from a simple PL experiment without the necessity of measuring, e.g., the second-order intensity correlation function to identify laser action in high- $\beta$ microlasers.

In this work, we report on combined experimental and theoretical studies of high-quality QD-micropillar cavities in both the single- and multiemitter regime by using a unique $\mu \mathrm{PL}$ setup that allows us to simultaneously access axial and lateral emission. Moreover, we can apply lateral excitation of the micropillars to avoid reflection of light within the stopband of the resonator. This is very beneficial for strictly resonant and quasiresonant (e.g., $p$-shell) excitation schemes. Lateral excitation also minimizes excitation-induced local heating and dephasing, as much lower excitation power is required to reach the same system occupation compared to excitation through the top facet of the micropillar. In our experiment, lateral detection is first used in the multiemitter regime to monitor the lateral losses and to determine their dependence on the pillar diameter. These experimental results are compared with numerical results of finite-element (FEM) numerical simulations [27-30], which allow us to describe intrinsic lateral losses due to the leaky modes as a function of the micropillar diameter. Furthermore, detecting emission in the axial and lateral directions is used to analyze the correlation between emission into the cavity mode and into the leaky modes in the single emitter-single photon interaction regime of $\mathrm{CQED}$. Moreover, the ratio of the intensities detected in the axial and lateral directions reflects the directionality of emission as well as the relative contributions from spontaneous and stimulated emission. The intensity ratio has been proposed, experimentally proven, and modelled theoretically (within the microscopic laser model utilizing the cluster expansion method [31]) to be a sensitive indicator of laser action. Such a measure is of great importance for the study of high- $\beta$ microlasers, for which the characteristic $S$ shape is no longer clearly visible in the input-output curve [24].

The paper is organized as follows. In Sec. II, the investigated sample and our experimental setup are described. Experimental results and corresponding theoretical modeling are presented in Sec. III. The discussion of the results is divided into three sections. Section III A addresses the lateral losses and their dependence on the micropillar diameter. In Sec. III B single-QD cQED phenomena are studied in both weak and strong light-matter coupling regime. Finally, Sec. IIIC is devoted to studies of high- $\beta$ microlasers and introduces the directionality of the emission as a sensitive indicator of lasing action. Section IV concludes the paper.

\section{EXPERIMENTAL}

The spectroscopic studies were performed on high-quality micropillar cavities based on AlAs/GaAs microresonators with a single layer of self-assembled $\mathrm{In}_{0.4} \mathrm{Ga}_{0.6}$ As QDs as an active medium. Micropillars with diameters in the range of

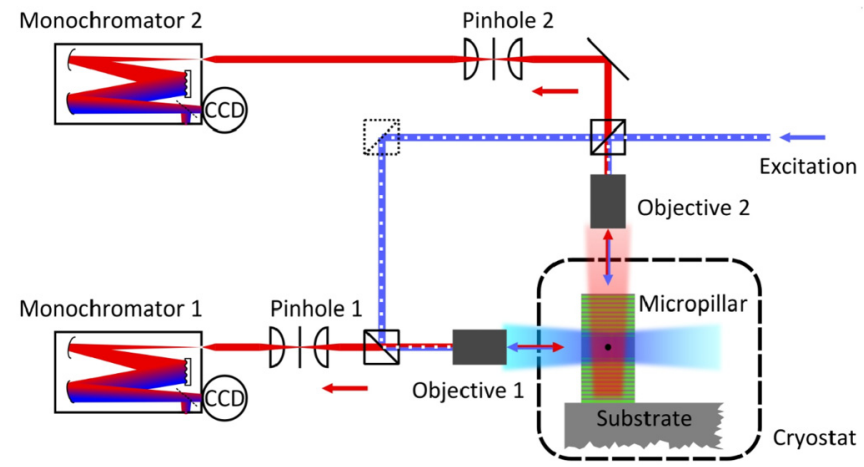

FIG. 1. (Color online) Schematic view of the experimental configuration. The sample is mounted onto the cold finger of a He-flow cryostat and can be accessed in the lateral and axial directions by two independent microscope objectives. Single micropillars under study can be excited optically in either the axial or lateral direction (blue dotted beam path) and the photoluminescence signal can be simultaneously detected in both directions (red solid beam path) via spectrometers 1 and 2 .

1.6 to $4.0 \mu \mathrm{m}$ were realized by high-resolution electron-beam lithography followed by plasma etching in a nondeterministic technology [32]. Lateral access to the micropillars is enabled by a precise cleaving of the sample, so dense arrays of micropillars (approximately $10-\mu \mathrm{m}$ distance between adjacent pillars) are located at the edge of the sample. For details of the sample layout and the processing see Ref. [33]. In order to obtain comprehensive insight into the emission features of the QD-micropillars we developed an experimental setup which is capable of exciting the structures either in the axial or the lateral direction and to detect emission simultaneously in both directions by two independent spectrometers. The setup is schematically shown in Fig. 1. The sample is mounted on the cold finger of the He-flow cryostat, enabling temperature-dependent measurements in the range of 4 $340 \mathrm{~K}$. Additionally, the cryostat is equipped with a customized top cover featuring two windows which enable optical access from the side and the top. The sample can be excited in the lateral or the axial direction via microscope objectives 1 or 2 , respectively. In the case of all experimental results presented here, the lateral excitation (perpendicular to the pillar axis) was used. Both detection paths include a $0.75-\mathrm{m}$-focal-length spectrometer equipped with a Si-based charge-coupled-device detector. The spectrometers can be controlled independently for simultaneous detection of signals in the lateral and axial directions with a spectral resolution of $25 \mu \mathrm{eV}$. The microscope objectives with a numerical aperture of 0.4 are attached to $x-y-z$ nanopositioners, which allow for a precise computer-controlled positioning and automatized adjustment of both excitation and detection paths. Additionally, a pinhole configuration is used in the axial detection path in order to filter out the stray light. In the case of lateral detection such a spatial filtering cannot be effectively applied, because the cavity mode stray light originating directly from scattering losses at the sidewalls of the micropillar coincides spatially with the leaky-mode emission. All experimental data presented in this work were obtained at temperatures between $8 \mathrm{~K}$ and $30 \mathrm{~K}$ under nonresonant excitation with a frequency-doubled continuous-wave $\mathrm{Nd}$ :YAG laser at $532 \mathrm{~nm}$. 

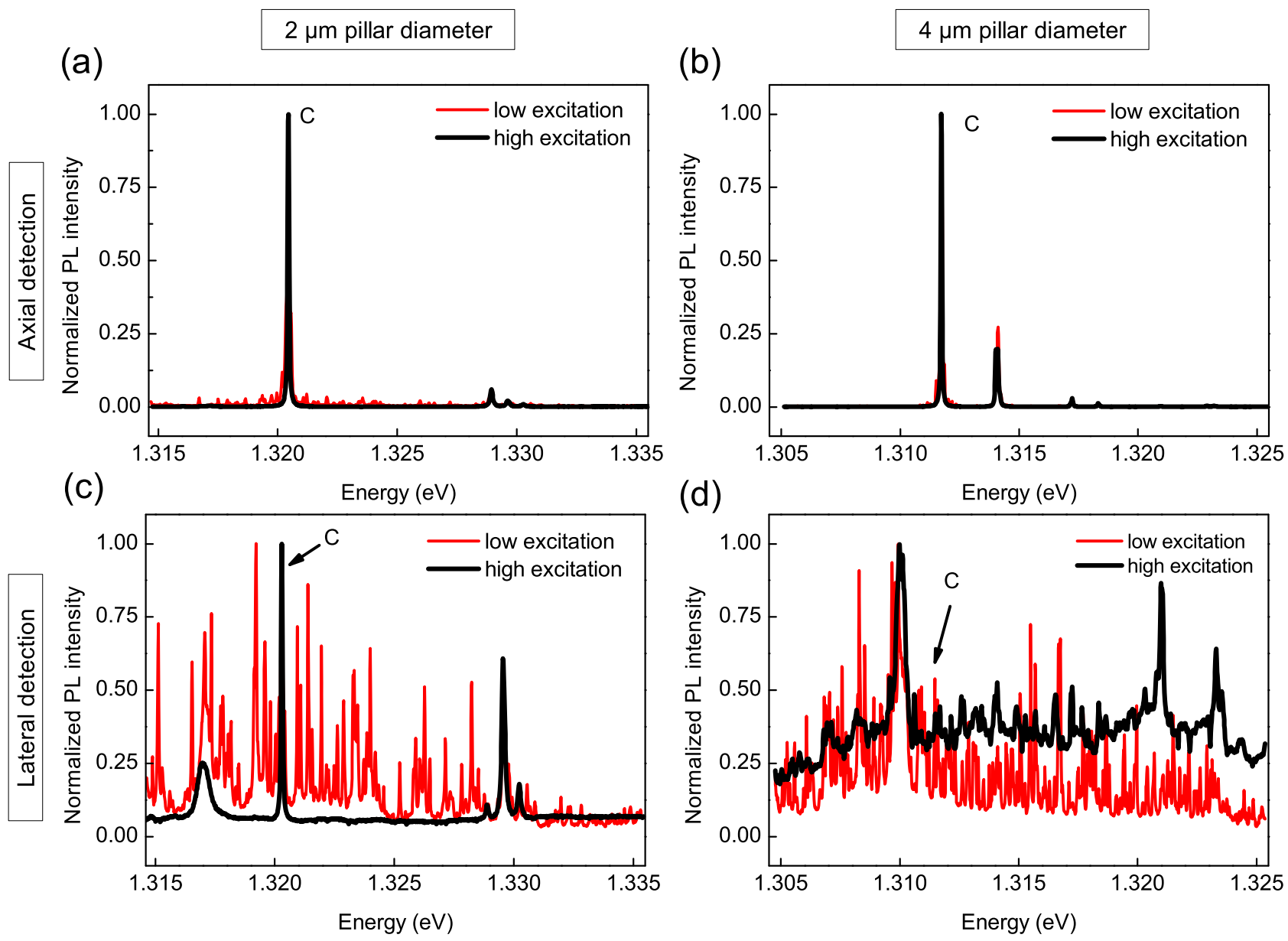

FIG. 2. (Color online) Comparison of $\mu$ PL spectra for axial [(a) and (b)] and lateral [(c) and (d)] detection for two pillar diameters $d_{c}=2.0 \mu \mathrm{m} \mathrm{[(a)} \mathrm{and} \mathrm{(c)]} \mathrm{and} 4.0 \mu \mathrm{m}[(\mathrm{b})$ and (d)]. The spectra were recorded under lateral excitation at two different excitation conditions: excitation power equal to half of the saturation power ("low excitation," thin red traces) and at saturation power ("high excitation," thick black traces) of the QDs emission, respectively. Using axial detection [(a) and (b)], the fundamental cavity mode (C) clearly dominates the spectra, while pronounced QD-related emission lines are visible in the emission detected in the lateral direction [(c) and (d)]. Emission of the cavity mode can hardly be observed under lateral detection in the case of the 4.0- $\mu \mathrm{m}$ micropillar (d). It is much stronger (in comparison to the QD signal) for the smaller micropillar with $d_{c}=2.0 \mu \mathrm{m}$ (c) and clearly dominates the spectrum at high excitation, which is not the case for larger micropillars. This nicely reflects the presence of enhanced lateral losses in the small-diameter regime.

\section{RESULTS AND DISCUSSION}

In the following we apply our detection scheme to study and differentiate between lateral and axial photoluminescence of QD-micropillars with various diameters. The first section deals with a diameter-dependent analysis of lateral emission of the fundamental cavity mode which gives insight into the respective lateral-photon-loss channels. Then we address correlations between lateral and axial emission in the weak and strong coupling regimes of cQED. In the final part we investigate the excitation power dependence of lateral and axial emission to establish a further criterion for the onset of lasing in high- $\beta$ microlasers.

\section{A. Diameter dependence of lateral emission: Addressing lateral-loss channels}

Our experimental configuration provides the appealing to study the optical loss channels of micropillar cavities and, in particular, to address the diameter dependence of sidewall losses. For a qualitative comparison Fig. 2 shows $\mu \mathrm{PL}$ spectra detected in the axial [Figs. 2(a) and 2(b)] and lateral [Figs. 2(c) and 2(d)] directions for two micropillars with a diameter $d_{c}$ of $2.0 \mu \mathrm{m}$ [Figs. 2(a) and 2(c)] and $4.0 \mu \mathrm{m}$ [Figs. 2(b) and 2(d)], respectively. The micropillars were excited in the lateral direction with an excitation power corresponding to about half the QDs emission saturation pump power (thin red traces) and to the saturation pump power (thick black traces), respectively. We observe distinct features in the emission from the large micropillar with $d_{c}=4.0 \mu \mathrm{m}$ : In the axial direction the fundamental cavity mode $\mathrm{C}$ dominates the emission spectrum and QD related lines are hardly visible [cf. Fig. 2(b)]. In contrast, the cavity mode is very weak in lateral emission, independent of the excitation conditions, while strong emission from single QDs can be observed in this configuration [Fig. 2(d)] . These observations nicely reflect that emission from off-resonant QDs is suppressed in axial direction by the cavity's stopband and that lateral losses are of minor importance for large diameter micropillars [34]. The situation differs strongly for the micropillar with $d_{c}=2.0 \mu \mathrm{m}$ 
for which significant lateral losses lead to a pronounced contribution of the cavity mode also in lateral emission (c). We would like to note that the strong PL background signal in Fig. 2(d) is related to ensemble emission of about 500 QDs in the active layer of the micropillar (approximately 125 QDs for the $2.0-\mu \mathrm{m}$ micropillar).

In order to investigate the diameter dependence of lateral losses in more detail, we systematically studied the lateral emission of micropillars with $d_{c}$ in the range of 1.7 and $4.0 \mu \mathrm{m}$. In particular, for each micropillar the integrated intensity of the cavity mode is set into relation to the integrated intensity of QD emission at saturation (both measured in the lateral direction) in a fixed spectral range. By this normalization we establish a rather precise measure of the diameter-dependent lateral emission intensity of the cavity mode. We have chosen this approach because the absolute intensity of lateral cavity emission depends very sensitively on the adjustment of the collection optics. To verify the applied normalization method with respect to that for selected QDmicropillars, input-output curves were recorded multiple times for both detection directions under slightly different alignment conditions. A quantitative comparison of the normalized data proved the consistency of the obtained results and therefore the validity of the normalization procedure and robustness of our results and, as a consequence, derived conclusions against the alignment of the optical system. Also, the micropillars with nominally the same diameters are not exactly identical, e.g., with respect to the $Q$ factors. Moreover, the structural defects at the sidewalls as well as other scattering centers are randomly distributed and strongly influence the absolute intensity values. The associated normalized emission intensity of the cavity mode detected in the lateral direction is plotted in Fig. 3 as a function of the pillar diameter. Each experimental data point presents a statistical average of five micropillars

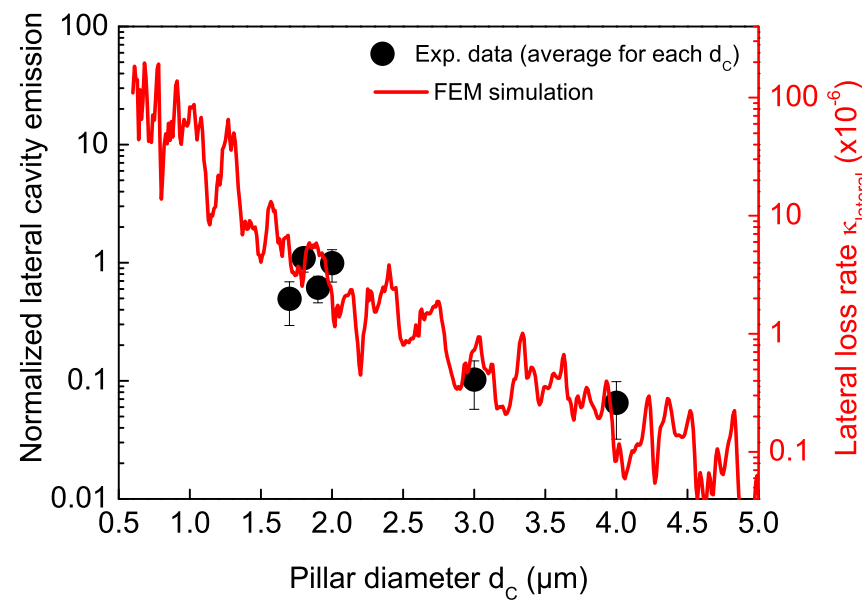

FIG. 3. (Color online) Diameter dependence of the lateral losses. The experimental data (black dots) presents the lateral intensity of the cavity mode signal normalized to the lateral emission of the QDs at saturation. The increase of this intensity in the small-diameter range is a clear signature of enhanced overall lateral losses (sum of intrinsic and sidewall losses). This experimental observation is in agreement with FEM numerical simulations (red trace) showing enhanced intrinsic lateral losses at small pillar diameters.
(10 pillars in the case of $2-\mu \mathrm{m}$ pillars) and the corresponding uncertainty is given by the standard deviation (no experimental error included). The reason for the spread in the experimental data can be twofold: (i) slightly different experimental conditions/detection efficiency due to imperfect optical alignment and (ii) differences in the actual QD-micropillar systems. Our normalization procedure minimizes the first effect and, therefore, we can conclude that the second effect is the main source contributing to the error bars. It is clearly seen that, while lateral losses, proportional to the normalized emission intensity in the lateral direction, are of minor importance for large diameters, they become significant for diameters below about $3.0 \mu \mathrm{m}$, as expected in the small-diameter regime. Interestingly, there are large variations in the experimental data in the low-diameter range. In order to obtain more insight into the dependence of the lateral losses on the pillar diameter, we performed numerical calculations using the commercial finite-element-method solver JCMSUITE by JCMwave.

The micropillars' fundamental optical mode has been calculated by solving the eigenvalue problem of the timeharmonic Maxwell equations. For details on this method we refer the interested reader to Ref. [35]. Simulations have been carried out using finite elements of third degree and three iterative refinement steps for the triangular mesh. The geometry of the micropillar was modelled in a rotational symmetric setting. The mean layer thickness of the DBR mirror pairs (GaAs: $67.5 \mathrm{~nm}$; AlAs: $86.5 \mathrm{~nm}$ ) and the GaAs cavity $(296 \mathrm{~nm})$ was determined from scanning electron microscopy images of a cleaved planar sample part of the same wafer. Additionally, the finite etching depth of 10 mirror pairs remaining in the lower DBR was taken into account. Taking the solutions of the eigenmode problem for the fundamental optical mode of an ideal cavity (without any structural imperfections), we calculated the total diameter-dependent loss rate by the relation $\kappa\left(d_{c}\right)=2 \operatorname{Im}(\omega) / \operatorname{Re}(\omega) \propto 1 / Q$, where $\operatorname{Im}(\omega)$ and $\operatorname{Re}(\omega)$ denote the imaginary and real part of the eigenvalue of the resonance frequency $\omega$. To describe the experimentally determined ratio introduced above, we assume that the total loss rate of the micropillar system can be described by $\kappa\left(d_{c}\right)=\kappa_{\text {axial }}+\kappa_{\text {lateral }}\left(d_{c}\right)$ with $\kappa_{\text {axial }}$ being the diameter-independent loss rate through the upper DBR given in the planar cavity limit $\left[\kappa_{\text {axial }}=\kappa_{2 D}=\kappa\left(d_{c} \rightarrow \infty\right)\right]$ and $\kappa_{\text {lateral }}$ describing the intrinsic lateral-loss rate. Given this, the diameter-dependent lateral losses of an ideal cavity can be calculated as $\kappa_{\text {lateral }}\left(d_{c}\right)=\kappa\left(d_{c}\right)-\kappa_{2 D}$.

The calculated lateral losses are plotted in Fig. 3 as a function of the pillar diameter (red trace). The simulation confirms an increase in lateral losses with decreasing diameter. It is important to note that only intrinsic lateral losses are included in the simulations, while both intrinsic and structural sidewall losses contribute to the measured lateral emission. Consequently, there should be a systematic difference between measured and calculated lateral losses corresponding to the additional contribution of sidewall losses present in the experiment. As we are interested only in the relative changes of the lateral losses as a function of the micropillar diameter, the theoretical dependence was scaled vertically to emphasize the respective correspondence. Good agreement between the simulations and experimental results indicates the same scaling of the diameter dependence of the emission 

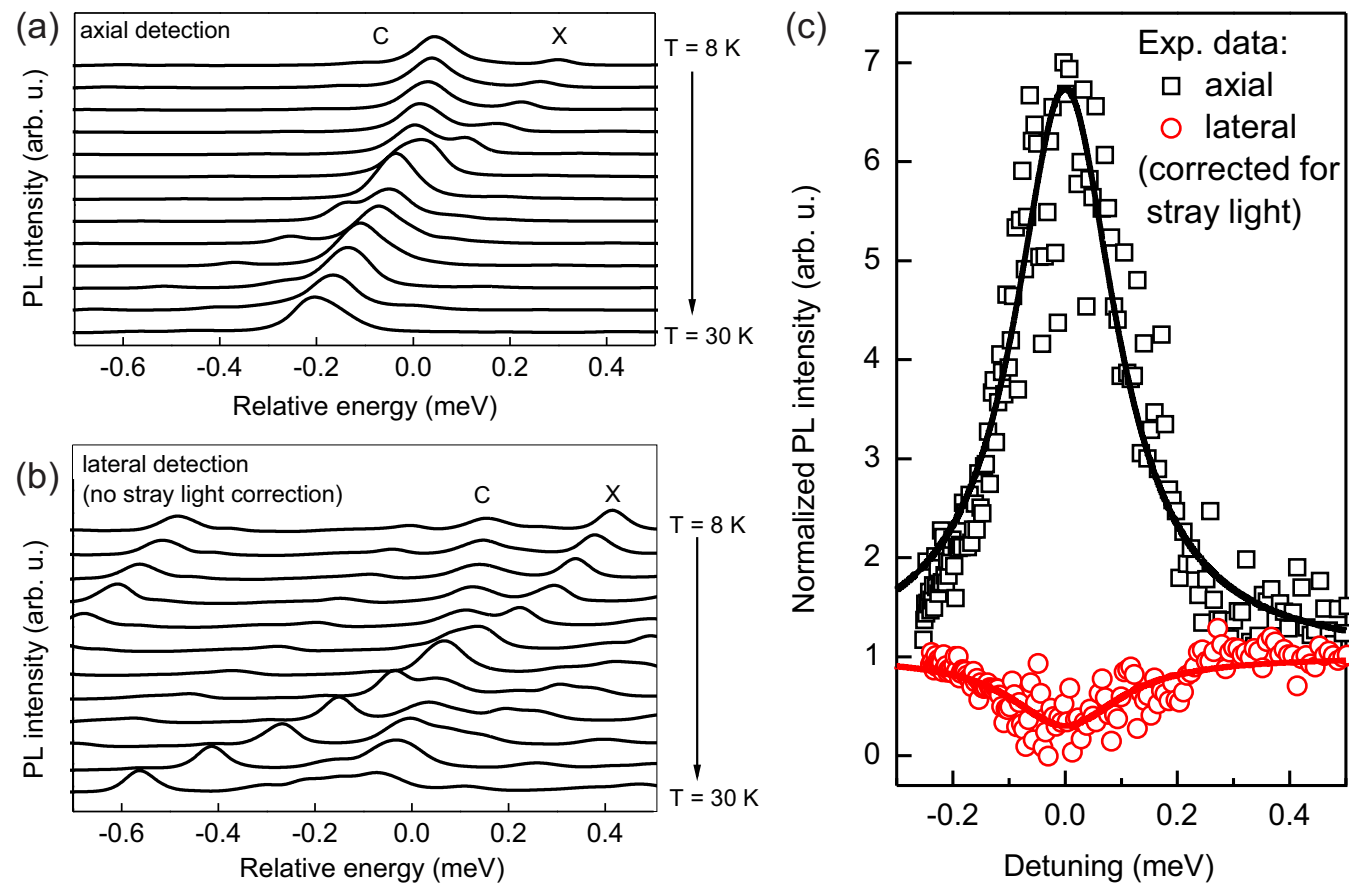

FIG. 4. (Color online) Study of a weakly coupled QD-micropillar system using axial (a) and lateral (b) detection. Temperature-dependent spectra show a crossing of the QD exciton emission line $(\mathrm{X})$ and the fundamental cavity mode $(\mathrm{C})$ which is characteristic for the weak coupling regime. The investigated micropillar has a diameter of $2.0 \mu \mathrm{m}$ and a $Q$ factor of 18000 . Pronounced enhancement of the QD emission on resonance due to the Purcell effect can be seen only in axial emission (a). The normalized integrated intensities of the QD in axial and lateral (corrected for the stray light) emission are plotted in (c) and show a pronounced anticorrelation as expected from Eqs. (1) and (2). Respective fits allow us to extract Purcell factors of $F_{P, C}=8.1 \pm 2.1$ (black upper trace) and $F_{P, L}=6.7 \pm 0.7$ (red lower trace), respectively.

into the leaky modes and sidewall scattering. This is a result of the fact that rates of both loss channels are determined by the electric field amplitude at the edge of the micropillar which is proportional to $1 / d_{c}$. Moreover, pronounced oscillations of the lateral-loss rate can be observed in the small diameter regime in accordance with previous studies [36], in which associated $Q$-factor oscillations were explained in terms of an impedance mismatch between the guided mode of the central cavity layer and the associated bloch modes of the top and bottom mirrors. Thus, the results of numerical simulations not only describe well the overall trend of enhanced lateral losses for small diameters but also provide a possible explanation for the strong variation of the experimental data in this regime which qualitatively recalls the known effect of diameter-dependent $Q$-factor oscillations [36].

\section{B. Single-QD cQED effects observed in axial and lateral emission}

We will now focus on single-QD-single-photon coupling effects in the cQED regime. Here, our combined lateral and axial detection scheme gives us the appealing opportunity to study the interplay between emission into the axially emitting cavity mode and lateral emission into the leaky modes at the single emitter level. This interdependence is described by the $\beta$ factor which expresses the probability of coupling a photon emitted by a single QD into the cavity mode. This probability is usually approximated by $\beta=F_{P} /\left(F_{P}+1\right)$ [37] and depends on the spectral detuning $\Delta=E_{X}-E_{C}$ between the QD exciton $(\mathrm{X})$ and the cavity mode $(\mathrm{C})$ via the Purcell factor according to $F_{P}(\Delta) \propto\left(\gamma_{C} / 2\right)^{2} /\left[\left(\gamma_{C} / 2\right)^{2}+\Delta^{2}\right]$ with the cavity mode full width at half maximum, $\gamma_{C}$.

Moreover, since the QD exciton decay is restricted to a single photon at a given time, one expects a pronounced anticorrelation between intensity of emission into the cavity mode $I_{C}$ proportional to $\beta$ and emission into the leaky modes $I_{L}$ proportional to $1-\beta$. In our system we studied the cQEDcontrolled emission into the cavity mode and into the leaky modes, respectively, via temperature-dependent single-QD measurements in the weak as well as in the strong light-matter coupling regime.

At first, we analyzed a weakly coupled QD-micropillar using our combined lateral-axial detection scheme and the temperature as a tuning parameter. The corresponding series of spectra recorded at about $10 \%$ of the saturation pump power of the QD X are depicted in Fig. 4 for axial [Fig. 4(a)] and lateral [Fig. 4(b)] detection, respectively. The exciton emission is hardly visible in the axial direction when the QD is off-resonant with the cavity mode and we observe strong enhancement of emission when the single QD is tuned through resonance with the cavity mode under variation of the temperature between 8 and $30 \mathrm{~K}$. The situation differs in the case of lateral emission, where the QD exciton is clearly visible and surpasses the cavity mode intensity in the whole tuning range. On resonance, there is a slight increase in intensity, which is at first sight against our expectation that emission into the cavity mode and into the leaky modes should be anticorrelated. However, one needs to take into account that lateral emission includes not only light coupled into the leaky modes but also the stray light from the cavity mode, as discussed above. 
Therefore, in order to distinguish between the two components, we normalized the lateral emission with the emission in the axial direction under the assumption that the sidewall scattering losses are independent of the detuning and that the stray light contributing to the laterally detected emission is proportional to the emission in the axial direction. In fact, the sidewall scattering losses should only depend on the structural imperfections of the micropillar. In the next step, we determined the intensity of the QD excitonic emission into the cavity mode and into the leaky modes, respectively, which are plotted in Fig. 4(c) as a function of the detuning $\Delta$. The competition between emission into both types of modes can nicely be described in terms of the detuning-dependent cQED effects in the weak coupling regime: Due to an enhanced local density of optical states on resonance the emission of the QD is preferentially coupled into the cavity mode according to

$$
I_{X->C} \propto \beta(\Delta)=F_{P}(\Delta) /\left[F_{P}(\Delta)+1\right],
$$

while emission into the leaky modes is given by

$$
I_{L} \propto 1-\beta(\Delta)=1-F_{P}(\Delta) /\left[F_{P}(\Delta)+1\right] .
$$

Accordingly, the emission intensity $I_{X->C}$ increases and shows a maximum on resonance $(\Delta=0)$ where a minimum of $I_{L}$ is expected to occur. The described detuning dependence of each contribution $I_{X->C}$ and $I_{L}$ allows us to determine the Purcell factor independently by fitting the corresponding dependencies plotted in Fig. 4(c) by Eqs. (1) and (2), respectively. The experimental data are nicely described by the theory and fitting yields Purcell factors $F_{P, C}=8.1 \pm 2.1$ [black upper trace in Fig. 4(c)] and $F_{P, L}=6.7 \pm 0.7$ [red lower trace in Fig. 4(c)], which are in good quantitative agreement within the estimated fitting errors. We would like to note that this method of extracting the Purcell factor is in general more reliable than fitting only emission in the axial direction as performed, e.g., in Ref. [6], because it provides two independent measures of the Purcell factor by considering both the detuning dependence of emission into the cavity mode and into the leaky modes. Each of them involves only one fitting parameter, namely the Purcell factor itself. However, the theoretically estimated maximal achievable Purcell factor resulting from effective mode volume and $Q$ factor of the microcavity for the investigated pillar equals about 60 . It is almost 10 times larger than the experimentally determined one. Even though the achievable Purcell factor might be overestimated due to operation conditions very close to the strong coupling regime [38], there are also physical effects lowering the Purcell factor, in particular, spatial mismatch between the QD position in the cavity and the maximum of the electromagnetic field distribution of the fundamental mode and dipole orientation mismatch between the QD and the cavity mode reducing the coupling strength. It is well established that in the case of the investigated structure the QD polarization axes are mostly oriented along [1-10] and the [110] crystallographic direction [39], whereas the deformation of the nominally circular in cross-section micropillars leads to linear polarization of the cavity modes along the [100] and [010] directions [32] which means that the respective dipoles are oriented at a $45^{\circ}$ angle which can additionally lower the achievable Purcell factor by a factor of 2 [40].
Beyond the weak coupling regime, which is governed by dissipative losses, it is instructive to study also the strong coupling regime using combined axial/lateral detection. In this coherent interaction regime of cQED, the spontaneous emission process becomes reversible and entangled light-matter states are formed, as described by the Jaynes-Cummings model $[41,42]$. In this context the question appears whether the formation and presence of such zero-dimensional polaritonic states has a significant impact on the relative coupling of light into the leaky modes and the cavity mode, respectively. In order to address this question, we performed temperature-dependent single-QD measurements, using the lateral and axial detections, for another micropillar with $d_{c}=2.0 \mu \mathrm{m}$, for which the strong coupling conditions are fulfilled. The corresponding waterfall plots for the axial and lateral emissions are depicted in Figs. 5(a) and 5(b). The characteristic anticrossings of the QD X and cavity mode are visible in both series of spectra and a vacuum Rabi splitting of $72 \mu \mathrm{eV}$ was extracted from the corresponding energy dispersion presented in Fig. 5(c). In the case of the coherently interacting QD-microcavity system in the strong coupling regime we can no longer distinguish between the excitonic and photonic component of the emission due to formation of entangled light-matter states. Thus, we applied the analogous analysis as for the strong coupling case but normalized and evaluated the combined intensity of the coupled QD-cavity system $\left(I_{C+X}\right)$, as opposed to the weak coupling case, in which the QD emission has been normalized separately and independently from the cavity emission. The result is depicted in Fig. 5(d) and does not show any characteristic anticorrelation between axial and lateral detection, in strong contrast to the weak coupling case discussed above. This is a clear signature of the coherent nature of the single-X-single-photon interaction in the strong coupling regime which does not allow one to distinguish between the bare uncoupled states and their decay channels anymore. The apparent increase of the integrated intensity as a function of detuning (temperature) can be related to the different temperature behavior of the emission between the QD and the cavity mode which was not canceled out due to joint normalization of the two branches. It is also interesting to note that the corresponding $\beta$ factor is close to unity in the strong coupling regime [43] which essentially means that emission from the resonant QD into the leaky modes is negligible. In fact, emission into the leaky modes would lead to a central peak in the emission spectrum [44] and, as a result, an anticorrelation between axial and lateral emission intensity on resonance which is not the case in our experimental data. Further studies in this regime could focus on resonance fluorescence of strongly coupled QD-microcavity systems including selective excitation of the QD or population of the cavity mode in a combined axial/lateral excitation scheme advantageous in view of efficient suppression of optical excitation due to spatial separation of the laser and signal [45].

\section{Excitation power-dependent studies of QD-micropillars}

In this section we address the excitation power dependence of the emission into the axial and lateral directions and its relation to laser action in high- $\beta$ QD-micropillar lasers 

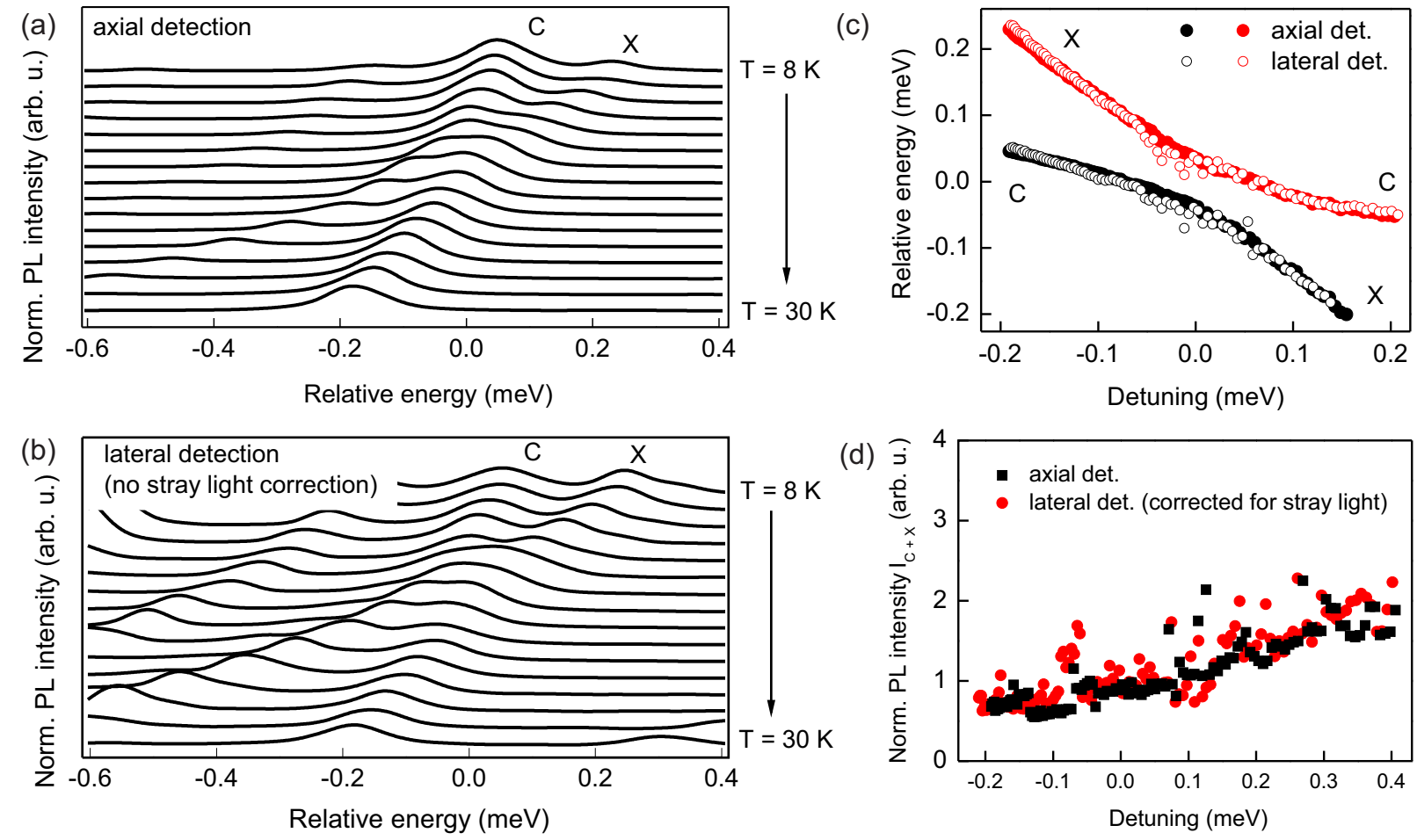

FIG. 5. (Color online) Study of a strongly coupled QD-micropillar system using axial (a) and lateral (b) detection. The micropillar has a diameter of $2.0 \mu \mathrm{m}$ and a $Q$ factor of 16000 . [(a) and (b)] Temperature-dependent emission spectra detected in the axial (a) and in lateral directions (b). In both cases an anticrossing of the QD exciton (X) and the cavity mode (C) can be observed in the respective energy dispersions (c) from which a vacuum Rabi splitting of $72 \mu \mathrm{eV}$ was determined. (d) Normalized sum of emission intensities of C and X detected in the axial (black squares) and lateral (red dots) directions. In contrast to Fig. 4(c), no anticorrelation between emission in the axial and lateral directions as a function of detuning $(\Delta)$ can be observed.

providing important insight into the relation between stimulated and spontaneous emission. The figure of merit in the analysis is the ratio $r$ of light (integrated emission intensity) detected in axial and lateral detection, $r=I_{\text {axial }} / I_{\text {lateral }}$, which is of particular interest as a measure of the directionality of emission. This directionality should increase at threshold due to the onset of stimulated emission, and we expect this ratio to be a good indicator of laser action. This is important especially for high- $\beta$ microlasers, for which it becomes more and more difficult to prove the onset of lasing when $\beta$ approaches unity and the typical s shape can no longer be clearly observed in the input-output curve [cf. Fig. 6(a)].

The excitation power dependence of emission in the axial and lateral directions was investigated for a micropillar laser with $d_{c}=2.0 \mu \mathrm{m}$ for which high $\beta$ values are expected due to limited number of emitters in the active region and a low mode volume. Figure 6(a) shows the emission intensity detected in the axial $I_{\text {axial }}$ (open squares) and lateral directions $I_{\text {lateral }}$ (open circles) as a function of excitation power measured outside the cryostat in a double-logarithmic scale. The axial/lateral traces are normalized with respect to each other to give $r=1$ at low excitation power. This accounts for different setup efficiencies in the detection paths, as well as for the fact that only a part of the lateral emission is detected and also for a nonzero contribution of the stray light to the lateral emission. The axial emission shows the expected S-shaped dependence on excitation power $P$ with a smooth transition from spontaneous to stimulated emission around the threshold at $P \approx 100 \mu \mathrm{W}$, well known for high- $\beta$ microlasers [46]. Below threshold, both axial and lateral emission intensity traces increase similarly with pump power, because the stimulated emission into lasing - as well as into nonlasing modes - is proportional to carrier inversion. Emission into nonlasing modes is expected to saturate above threshold, as stimulated emission depletes the inversion. This effect is actually masked by stray light from the lasing mode scattered into the lateral direction. A close examination of the input-output curves reveals, however, a stronger increase of $I_{\text {axial }}$ compared to $I_{\text {lateral }}$ at the onset of stimulated emission, indicating that more stimulated emission is directed into the axially emitting laser mode. A quantitative measure of the directionality is the ratio $r$ introduced above. The excitation-power dependence of this parameter is depicted in Fig. 6(b). Due to the applied normalization procedure, $r$ is close to unity at low excitation powers and increases quickly around threshold ( $P \approx 100 \mu \mathrm{W}$ ) up to about 1.8 .

The above interpretation is confirmed by calculations within a microscopic laser model, describing the coupling between an ensemble of $N$ identical multilevel QD emitters to a high- $Q$ laser mode via a Jaynes-Cummings-like dipole Hamiltonian $H_{D}=g\left(b^{\dagger}+b\right) D_{s}$, coupling the dipole moments $D_{s}=X_{s}^{\dagger}+X_{s}$ of the $N$ QDs to creation $\left(b^{\dagger}\right)$ and annihilation $(b)$ of a cavity photon with the coupling matrix element $g$. The dipole moment $D_{s}$ contains contributions from all carrier configurations in the confined QD states that allow for a carrier transition at the QD $s$ shells. Emission into nonlasing modes is taken into account by coupling the 
(a)

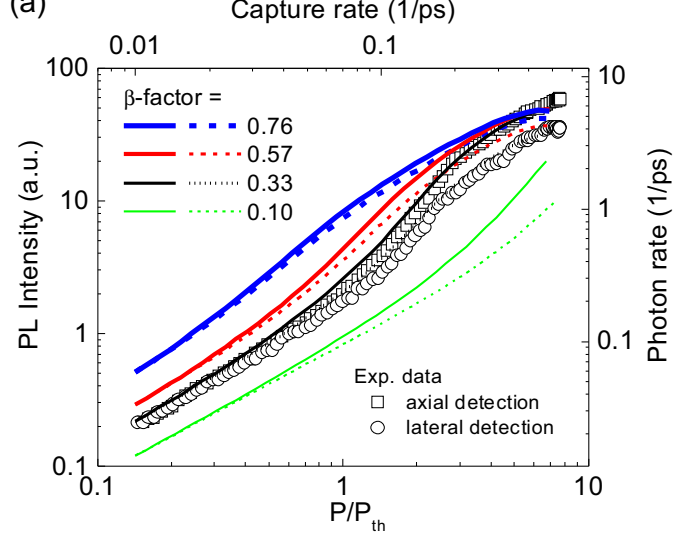

(b)

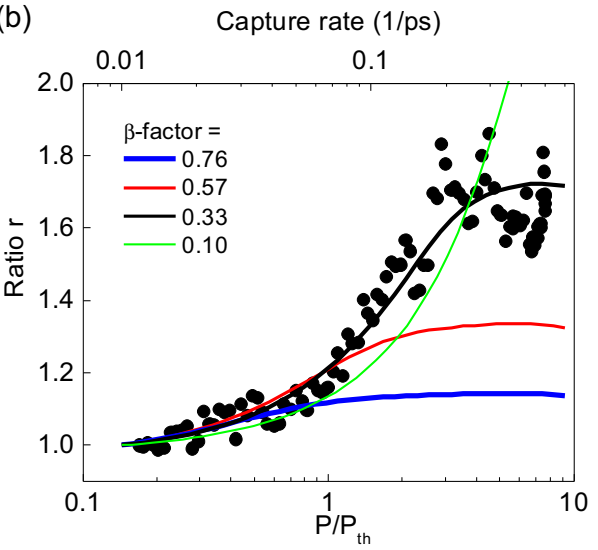

FIG. 6. (Color online) (a) Excitation-power dependence of the intensity of emission detected in the axial (open squares) and lateral (open circles) directions from a QD-micropillar with $d_{c}=2.0 \mu \mathrm{m}$ and $Q=15200$ at $T=10 \mathrm{~K}$. (b) Ratio $r$ of emission intensity detected in the axial and lateral directions as a function of excitation power. This ratio increases at threshold due to the onset of stimulated emission at about $100 \mu \mathrm{W}$, in agreement with the nonlinear increase of the intensity in panel (a) at the same excitation power. In both panels the experimental data (symbols) are well described by the theory (black solid lines/dashes) based on a cluster expansion method with $\beta=0.33, g=\kappa=0.15 / \mathrm{ps}$, $\Gamma_{r, 0}=1 / \mathrm{ps}, \Gamma_{r}^{\prime}=18 / \mathrm{ps}, \tilde{\Gamma}=19 \%$, and $\Gamma_{\mathrm{sp}}=\Gamma_{\mathrm{nl}}^{s}=\Gamma_{\mathrm{nl}}^{p}=0.150 / \mathrm{ps}$. Moreover, in additional theoretical traces colors and thicknesses of the solid (axial emission) and dashed lines (lateral emission) indicate the influence of the $\beta$ factor on the presented dependencies. The different $\beta$ values, $0.10,0.43,0.57$, and 0.76 , are associated with $\Gamma_{\mathrm{sp}}=0.375 / \mathrm{ps}, 0.112 / \mathrm{ps}, 0.075 / \mathrm{ps}$, and $0.0375 / \mathrm{ps}$, respectively.

emitter to the continuum of free-space modes in the Lindblad formalism at rate $\Gamma_{\mathrm{nl}}$, which ensures consistent dephasing of the laser transition introduced by the spontaneous emission. Further Lindblad-terms are used to account for $p$ - to $s$-shell carrier relaxation at rate $\Gamma_{r}$, capture of electron-hole pairs into each QD after barrier excitation at rate $P$, as well as the loss of cavity photons at rate $\kappa$, which is proportional to $1 / Q$.

The dynamics of the laser system is obtained from solving Heisenberg's equations of motion for quantum-mechanical expectation values for the mean photon number $\left\langle b^{\dagger} b\right\rangle$, the two photon expectation value $\left\langle b^{\dagger} b^{\dagger} b b\right\rangle$, the photon-assisted polarizations $\left\langle b^{\dagger} D_{s}\right\rangle$, and the electronic occupation probabilities for the different carrier configurations, such as the exciton. This approach is an advancement of the laser model first introduced in Ref. [47] with the consistent inclusion of scattering and dephasing processes treated within the Lindblad formalism [31,48].

To relate to the experimental data, we extract the axial emission rate $I_{\mathrm{axial}}=\kappa\left\langle b^{\dagger} b\right\rangle$ and the lateral emission rate $I_{\text {lateral }}=\Gamma_{\mathrm{nl}}^{s} f_{s}+\Gamma_{\mathrm{nl}}^{p} f_{p}+\tilde{\Gamma}\left\langle b^{\dagger} b\right\rangle$. The population factors $f$ are obtained by summing over all carrier configurations in the QDs that allow for $s$ - and $p$-shell transitions. The last term accounts for the coupling of a percentage $\tilde{\Gamma}$ of scattered light from the axial mode into the lateral detection (stray light). Both rates are normalized at low intensities to yield $r=1$. Axial and lateral input-output traces and the ratio $r$ are shown in Fig. 6 for a parameter set specified in the figure caption. Good agreement with the experimental data is obtained for a $\beta$ factor of 0.33 and $19 \%$ of the axial cavity mode emission coupled into the lateral detection.

The interesting effect of $r$ saturating and slightly decreasing at high excitation powers is attributed to excitation-induced dephasing of the lasing transition, which is a well-known effect in QD systems [49]. With increasing carrier density in the continuum of states, more final states for scattering processes are available, which leads to an increase of scattering efficiency and the corresponding dephasing. This effect is modeled by assuming a pump-dependent increase of the scattering rates as $\Gamma_{r}(P)=\Gamma_{r, 0}(P=0)+P^{2} \Gamma_{r}^{\prime}$.

The definition of the $\beta$ factor becomes conceptually difficult in QD-microlaser systems, where the scattering efficiency changes over the wide range of excitation powers. The reason is that the rate of spontaneous emission into the laser mode, defined as

$$
\frac{1}{\tau_{l}}=\frac{2|g|^{2}}{\kappa+\sum_{i} \Gamma_{i}},
$$

depends explicitly on all scattering and dephasing processes, denoted as the sum of $\Gamma_{i}$. A meaningful definition is nevertheless possible in the limit of vanishing pump rates. In this regime, carrier scattering is negligible, and the dominating dissipation processes are photon losses and spontaneous emission. Thus, we define $\beta$ as $\frac{1}{\tau_{l}} /\left(\frac{1}{\tau_{l}}+\frac{1}{\tau_{n l}}\right)$, with

$$
\frac{1}{\tau_{l}}=\frac{2|g|^{2}}{\kappa+\Gamma_{\mathrm{nl}}} .
$$

Theoretical results shown in Fig. 6 for selected values of $\beta$ were obtained by tuning the loss rate into nonlasing modes. Naturally, in the limit of $\beta \rightarrow 1, r \rightarrow 1$, as the only contribution is then from scattered light from the axial emission. As such the nonlinear increases of $r$ at the lasing threshold appears to be a good indicator for the identification of laser action in high- $\beta$ microlasers. Introducing this new measure is of great importance as it enables us to identify the onset of stimulated emission in the case of smooth input-output curve from PL study. That would be even more crucial for single QD lasers for which the determination of the threshold is troublesome. 


\section{CONCLUSION}

In conclusion, we report on optical studies on high-quality QD-micropillars using a $\mu \mathrm{PL}$ setup enabling both excitation and simultaneous independent detection of emission in two perpendicular directions. The proposed detection scheme is used to investigate the competition between QD emission into the cavity mode and into the leaky modes in various regimes of cQED_from single QD phenomena in weak and strong coupling up to multiemitter lasing. Monitoring the lateral emission provides direct access to the sidewall losses and probes the directionality of the spontaneous emission, which is not possible in a standard PL setup. This study confirmed the enhancement of the lateral losses for micropillar diameters smaller than $3.0 \mu \mathrm{m}$, in agreement with the results of FEM simulations.

The coupling strength between excitons and photons determines the interplay between emission coupled to the cavity mode and into the leaky modes. In the weak coupling regime we observed an anticorrelation due to an enhanced local density of optical states on resonance leading to preferential coupling into the cavity mode. Whereas in the strong coupling regime no such distinction can be made between different decay channels due to the coherent nature light-matter inter- action. Finally, we showed that our detection scheme can be used to directly probe the directionality of emission as well as the ratio between stimulated and spontaneous emission. Both strongly increase above the threshold, which makes the ratio between axial and lateral emissions a very good measure of the laser action, as we have demonstrated experimentally and proven theoretically using a microscopic laser model. Thus, the intensity ratio between axial and lateral emission turns out to be a clear and easily accessible indicator of the onset of stimulated emission in high- $\beta$ lasers which can be very beneficial for their further study and development.

\section{ACKNOWLEDGMENTS}

The authors acknowledge financial support by the German Research Foundation via Projects No. Ka2318/4-1 and No. Re2974/3-1, the SFB 787 "Semiconductor Nanophotonics: Materials, Models, Devices," and the State of Bavaria. A.M. appreciates support from the Polish Ministry of Science and Higher Education within the "Mobilnosc Plus" program. We thank M. Emmerling and A. Wolf for expert sample preparation, and T.H. acknowledges helpful discussions with S. Burger regarding the FEM simulations in Sec. III A.
[1] F. Jahnke, ed., Quantum Optics with Semiconductor Nanostructures (Elsevier, Amsterdam, 2012).

[2] P. Michler, Single Semiconductor Quantum Dots (Springer, Berlin, 2009).

[3] M. T. Rakher, Quantum Optics with Quantum Dots in Microcavities (ProQuest, Ann Arbor, 2008).

[4] E. del Valle Reboul, Ph.D. thesis, Universidad Autonma de Madrid, 2009.

[5] C. Kistner, K. Morgener, S. Reitzenstein, C. Schneider, S. Höfling, L. Worschech, A. Forchel, P. Yao, and S. Hughes, Appl. Phys. Lett. 96, 221102 (2010).

[6] C. Böckler, S. Reitzenstein, C. Kistner, R. Debusmann, A. Löffler, T. Kida, S. Höfling, A. Forchel, L. Grenouillet, J. Claudon et al., Appl. Phys. Lett. 92, 091107 (2008).

[7] A. Badolato, K. Hennessy, M. Atatüre, J. Dreiser, E. Hu, P. M. Petroff, and A. Imamoğlu, Science 308, 1158 (2005).

[8] J. P. Reithmaier, G. Sek, A. Löffler, C. Hofmann, S. Kuhn, S. Reitzenstein, L. V. Keldysh, V. D. Kulakovskii, T. L. Reinecke, and A. Forchel, Nature 432, 197 (2004).

[9] P. Michler, A. Kiraz, C. Becher, W. V. Schoenfeld, P. M. Petroff, L. Zhang, E. Hu, and A. Imamoglu, Science 290, 2282 (2000).

[10] J. Claudon, J. Bleuse, N. S. Malik, M. Bazin, P. Jaffrennou, N. Gregersen, C. Sauvan, P. Lalanne, and J.-M. Gérard, Nat. Photon. 4, 174 (2010).

[11] T. Heindel, C. Schneider, M. Lermer, S. H. Kwon, T. Braun, S. Reitzenstein, S. Höfling, M. Kamp, and A. Forchel, Appl. Phys. Lett. 96, 011107 (2010).

[12] A. Dousse, J. Suffczynski, A. Beveratos, O. Krebs, A. Lemaitre, I. Sagnes, J. Bloch, P. Voisin, and P. Senellart, Nature 466, 217 (2010).

[13] S. Noda, Science 314, 260 (2006).
[14] S. Strauf, K. Hennessy, M. T. Rakher, Y.-S. Choi, A. Badolato, L. C. Andreani, E. L. Hu, P. M. Petroff, and D. Bouwmeester, Phys. Rev. Lett. 96, 127404 (2006).

[15] S. Reitzenstein, T. Heindel, C. Kistner, A. Rahimi-Iman, C. Schneider, S. Höfling, and A. Forchel, Appl. Phys. Lett. 93, 061104 (2008).

[16] M. Nomura, N. Kumagai, S. Iwamoto, Y. Ota, and Y. Arakawa, Opt. Express 17, 15975 (2009).

[17] M. Nomura, N. Kumagai, S. Iwamoto, Y. Ota, and Y. Arakawa, Nat. Phys. 6, 279 (2010).

[18] M. Lermer, N. Gregersen, M. Lorke, E. Schild, P. Gold, J. Mørk, C. Schneider, A. Forchel, S. Reitzenstein, S. Höfling et al., Appl. Phys. Lett. 102, 052114 (2013).

[19] A. Kavokin, J. J. Baumberg, G. Malpuech, and F. P. Laussy, Microcavities (Oxford University Press, Oxford, 2007).

[20] P. Lalanne and J. P. Hugonin, IEEE J. Quant. Electron. 39, 1430 (2003).

[21] Y. Zhang and M. Loncar, Opt. Lett. 34, 902 (2009).

[22] M. Lermer, N. Gregersen, F. Dunzer, S. Reitzenstein, S. Höfling, J. Mørk, L. Worschech, M. Kamp, and A. Forchel, Phys. Rev. Lett. 108, 057402 (2012).

[23] S. Reitzenstein and A. Forchel, J. Phys. D: Appl. Phys. 43, 033001 (2010).

[24] Y. Yamamoto, S. Machida, and G. Björk, Phys. Rev. A 44, 657 (1991).

[25] J. Vuckovic, O. Painter, Y. Xu, A. Yariv, and A. Scherer, IEEE J. Quant. Electron. 35, 1168 (1999).

[26] G. S. Solomon, M. Pelton, and Y. Yamamoto, Phys. Rev. Lett. 86, 3903 (2001).

[27] L. W. Zschiedrich, S. Burger, R. Klose, A. Schaedle, and F. Schmidt, Proc. SPIE 5728, 192 (2005). 
[28] J. Pomplun, S. Burger, L. Zschiedrich, and F. Schmidt, Phys. Status Solidi B 244, 3419 (2007).

[29] S. Burger, L. Zschiedrich, J. Pomplun, and F. Schmidt, in Integrated Photonics and Nanophotonics Research and Applications (Optical Society of America, Massachusetts, 2008), p. ITuE4.

[30] J. Pomplun, S. Burger, F. Schmidt, A. Schliwa, D. Bimberg, A. Pietrzak, H. Wenzel, and G. Erbert, Phys. Status Solidi B 247, 846 (2010).

[31] M. Florian, C. Gies, F. Jahnke, H. A. M. Leymann, and J. Wiersig, Phys. Rev. B 87, 165306 (2013).

[32] S. Reitzenstein, C. Hofmann, A. Gorbunov, M. Strauß, S. H. Kwon, C. Schneider, A. Löffler, S. Höfling, M. Kamp, and A. Forchel, Appl. Phys. Lett. 90, 251109 (2007).

[33] A. Löffler, J. P. Reithmaier, G. Sęk, C. Hofmann, S. Reitzenstein, M. Kamp, and A. Forchel, Appl. Phys. Lett. 86, 111105 (2005).

[34] T. Rivera, J.-P. Debray, J. M. Gérard, B. Legrand, L. ManinFerlazzo, and J. L. Oudar, Appl. Phys. Lett. 74, 911 (1999).

[35] M. Karl, B. Kettner, S. Burger, F. Schmidt, H. Kalt, and M. Hetterich, Opt. Express 17, 1144 (2009).

[36] S. Reitzenstein, N. Gregersen, C. Kistner, M. Strauss, C. Schneider, L. Pan, T. R. Nielsen, S. Höfling, J. Mørk, and A. Forchel, Appl. Phys. Lett. 94, 061108 (2009).

[37] J.-M. Gérard, Top. Appl. Phys. 90, 269 (2003).

[38] G. Khitrova, H. M. Gibbs, M. Kira, S. W. Koch, and A. Scherer, Nat. Phys. 2, 81 (2006).
[39] A. Musiał, P. Gold, J. Andrzejewski, A. Löffler, J. Misiewicz, S. Höfling, A. Forchel, M. Kamp, G. Sęk, and S. Reitzenstein, Phys. Rev. B 90, 045430 (2014).

[40] Y.-S. Lee and S.-D. Lin, Opt. Express 22, 1512 (2014).

[41] E. Jaynes and F. Cummings, Proc. IEEE 51, 89 (1963).

[42] B. W. Shore and P. L. Knight, J. Mod. Opt. 40, 1195 (1993).

[43] D. Press, S. Götzinger, S. Reitzenstein, C. Hofmann, A. Löffler, M. Kamp, A. Forchel, and Y. Yamamoto, Phys. Rev. Lett. 98, 117402 (2007).

[44] K. Hennessy, A. Badolato, M. Winger, D. Gerace, M. Atature, S. Gulde, S. Falt, E. L. Hu, and A. Imamoglu, Nature 445, 896 (2007).

[45] S. Ates, S. M. Ulrich, A. Ulhaq, S. Reitzenstein, A. Löffler, S. Höfling, A. Forchel, and P. Michler, Nat. Photon. 3, 724 (2009).

[46] S. M. Ulrich, C. Gies, S. Ates, J. Wiersig, S. Reitzenstein, C. Hofmann, A. Löffler, A. Forchel, F. Jahnke, and P. Michler, Phys. Rev. Lett. 98, 043906 (2007).

[47] C. Gies, J. Wiersig, M. Lorke, and F. Jahnke, Phys. Rev. A 75 , 013803 (2007).

[48] H. J. Carmichael, Statistical Methods in Quantum Optics 1 (Springer, Berlin, 1999).

[49] M. Lorke, T. R. Nielsen, J. Seebeck, P. Gartner, and F. Jahnke, Phys. Rev. B 73, 085324 (2006). 